\title{
Application of Experimental Modal Analysis to Determine Damping Properties for Stacked Corrugated Boxes
}

\author{
Changfeng $\mathrm{Ge}^{1}$ and Steven Sutherland ${ }^{2}$ \\ ${ }^{1}$ Rochester Institute of Technology, 78 Lomb Memorial Drive, Rochester, NY 14623, USA \\ ${ }^{2}$ US Patent and Trademark Office, 600 Dulany Street, Alexandria, VA 22314, USA \\ Correspondence should be addressed to Changfeng Ge; cfgmet@rit.edu
}

Received 18 March 2013; Accepted 26 May 2013

Academic Editor: Jun Wang

Copyright (c) 2013 C. Ge and S. Sutherland. This is an open access article distributed under the Creative Commons Attribution License, which permits unrestricted use, distribution, and reproduction in any medium, provided the original work is properly cited.

Multilayer stacked corrugated packaging boxes are a common shipping mode in packaging distribution. This study discusses how to determine the damping properties of stacked corrugated boxes using experimental modal analysis (EMA). Prior to the calculation of damping properties, two MATLAB-based digital filters were applied to process the sampled original signals. Both the logarithmic method and the curve fit method are used to compute the multiple damping ratios from the acceleration and displacement response data after the excitation. The study found that the viscous damping and dry friction damping take place in a different time frame in stacked corrugated boxes. This study recommends using the curve fit method to determine damping properties for a multipledegree-of-freedom system such as stacked corrugated boxes.

\section{Introduction}

The corrugated box is one of the most common shipping containers. Corrugated board is a sandwich structure consisting of two paper faced liners and one medium paper corrugated liner. Unitized corrugated boxes are usually stacked on a single pallet or slip sheet in a tractor trailer for transport. The rough road surface, unbalanced tire system, and flexing of the trailer cause the unitized shipping units to experience vertical impact and vibration.

In order to effectively simulate a dynamic performance of stacked corrugated boxes during transport, a mixeddamping multiple-degree-of-freedom (MDOF) model needs to be considered, consisting of mass, stiffness, and damping. The dynamic response of multilayer stacked shipping units during the transport is a combined function of different damping modes of viscous damping and dry friction damping. Presently, the research related to stacked shipping units focuses on four different levels: (a) using dummy shipping unit without damping; (b) a linear MDOF with a viscous damping; (c) a single-degree-of-freedom system with viscous and friction damping; and (d) a linear MDOF with combined viscous damping and dry friction.
The corrugated research community does not often see articles that address the damping in the simulation model other than pieces of research conducted by forest product laboratories in USA. Between the early 1960s and early 1980s, a series of research studies were carried out in the forest products laboratories to simulate the viscous damping factors on stacked shipping units. Godshall $[1,2]$ applied the formula $\zeta=1 / 2 \operatorname{Tr}$ to calculate the viscous damping ratio where $\mathrm{Tr}$ is the transmissibility at resonance. After obtaining the vibration transmissibility through the FRF spectrum, the estimated viscous damping ratio ranges were determined. From the 23 vertical-stacked shipping units, the damping ratio was between 0.07 and 0.30 , of which 0.115 was the most common damping ratio for vertically stacked corrugated boxes. Urbanik [3] developed a three-degree-of-freedom analytical model for three-layer-stacked corrugated boxes simulating vibration during transport and developed the maximum transmission ability and amplification. Although the stacked corrugated box in Urbanik's study was an MDOF system and had some degree of nonlinearity, the individual box in the stack was regarded as a multilinear viscously damped spring-mass system. In his analytical model, the viscous damping coefficient was estimated as $30 \%$ of 
the critical damping coefficient $c=2 \sqrt{m k}$, where $m$ is the mass of the product and package and stiffness $k$ is linearized from the static compression test curves in his studies. Both Urbanik and Godshall confirmed that simple linear vibration theory can be utilized with the data such as the stiffness from compression tests and transmissibility through vibration tests to satisfactorily predict resonant frequencies of loadcontainer systems, but a vibration test is needed in order to get the critical damping coefficient.

Chen et al. [4] compared the shock response curve of a theoretical viscous damping model to an experimental derived shock response curve of a simplified single-degreeof-freedom corrugated box and cushion system and found that the curves did not match. Chen suggested that a frictionviscous damping model for both corrugated and foam cushionings should be added into the model simulation. Chen also proposed a new friction equation based on the conservation of energy and a hysteresis loop method.

Wang et al. [5, 6] proposed an inverse substructure method for a non-linear MDOF packaging system. The excitation to the base of a lab-based model was used in Wang's study to derive the joint parameters between the structures. In addition, Wang also proposed a 3-dimensional shock spectrum for a nonlinear MDOF packaging system, where the damping ratio was a critical component for prediction of the dynamic response. Peleg [7] developed a nonlinear MDOF model for unitized corrugated boxes stacked on the pallet and proposed an estimate calculation method for viscous damping coefficients and dry friction damping ratio by combining the viscous damping and dry friction damping together. The hysteretic loop method was used in his study to calculate the combined damping coefficients for both impact and vibration. The actual damping coefficient remains a complicated factor because the equipment used in the study constrains the computer simulation.

Considering both viscous and dry friction dampings in a simulation will bring the model more close and accurate to a real system. The damping coefficients from the previous bodies of literature were all computed through an FRF curve obtained from vibration testing. Vibration testing is a laboratory simulation from which the displacement/acceleration, amplification, and natural frequencies of stacked corrugated boxes are obtained easily without knowing the stiffness or damping ratio of tested corrugated boxes. If the purpose of the computer simulation is to provide an alternative tool to the vibration testing, the data used for simulation should not be derived from the vibration test itself, but another simple testing or calculation. In addition, a combined damping coefficient does not give the difference between a viscous damping and a dry friction damping, as well as their effect on the unitized load, respectively. Also, the damping coefficient of a corrugated box varies within a range, depending on the location of this particular box in the unitized packaging. Therefore, having a simple and practical way to determine damping properties of stacked corrugated boxes becomes important as the damping ratios can be channeled into the software for predicting the dynamic performance of unitized goods in distribution.

\section{Materials and Methods}

This study models 3-layer stacked corrugated boxes as a superposition of three-degree-of-freedom linear models and determines their damping properties on the site using experimental modal analysis (EMA). A roving hammer is used to excite the base floor on which the three corrugated boxes are stacked on top of each other. The collected response data were initially input into the digital filters with the intention to investigate if the viscous damping and dry friction damping occur in different time frames in this study. Both the logarithmic decrement method and the curve-fit method were applied to calculate the damping ratio.

2.1. Experimental Modal Analysis. Experimental modal analysis (EMA) was developed in the late 1970s to determine the dynamic properties of a structure. It is typically carried out by exciting a structure in one location using a hammer and measuring the responses at other locations of the structure. The responses can be measured in the form of displacement, velocity, or acceleration in a time domain. The responses in time domain can be computed by an oscilloscope or software such as MATLAB to obtain frequency response function (FRF), and the output $X(\omega)$ is charted. Frequency response function (FRF) can be represented by a matrix of differential equations $F(\omega)=H(\omega) X(\omega)$ where $F(\omega)$ is the exiting input, $X(\omega)$ is the response, and $H(\omega)$ represents the dynamic properties of the structure. Ge and Goodwin [8] applied this engineering method to determine natural frequencies of stacked corrugated boxes, which are in agreement with the vibration testing.

2.2. The Logarithmic Decrement Method. There are several methods to measure damping properties. They are the logarithmic decrement method in time domain, the step response method, the hysteretic loop method in time domain, and the magnification factor method and bandwidth method, both based on frequency domain $[7,9,10]$.

The logarithmic decrement method is the most popular method to measure the damping ratio for a single-linearviscous-damping model (a). Figure 2 shows a response $X(t)$ chart after the system is impacted by a roving hammer. The decay response of model can be expressed as $x(t)=$ $X e^{-\zeta \omega_{n} t} \sin \left(\omega_{d} t+\varphi\right)$ illustrated in Figure 2, where $X$ is the amplitude, $\zeta$ is the damping ratio, $\omega_{d}=\left[1-\zeta^{2}\right]^{1 / 2} \omega_{n}$ is the damped circular frequency, and $\varphi$ is the phase angle of damped oscillations.

In the chart, the decrement of the response can be determined by taking the natural logarithm of the first peak divided by the second peak of the oscillatory function. This number then could be used to determine the damping ratio and damping coefficient of the system. The peaks can be measured from an oscilloscope and transferred into a spreadsheet.

Peaks $A_{1}$ and $A_{2}$ in Figure 2 correspond to the numerator and denominator within the natural log function, respectively, in the following equation. $A * \sin \left(\omega_{d} t\right)$ is representative of a decaying sinusoidal function. The displacement at each 


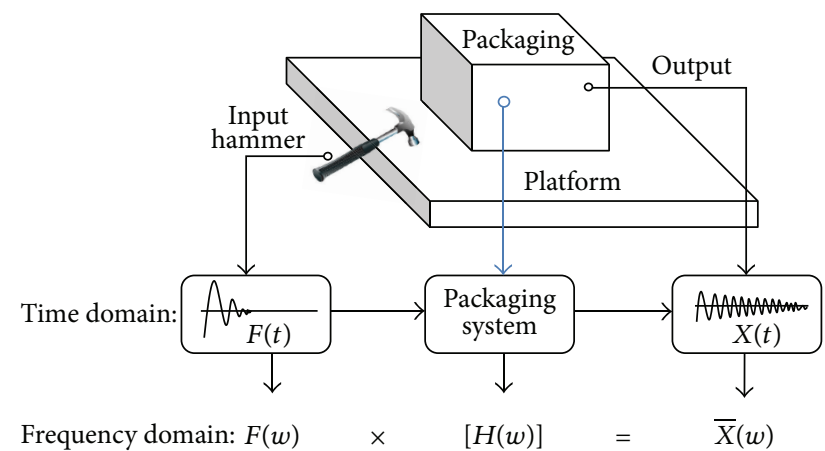

FIGURE 1: EMA method for a single-degree-of-freedom system.

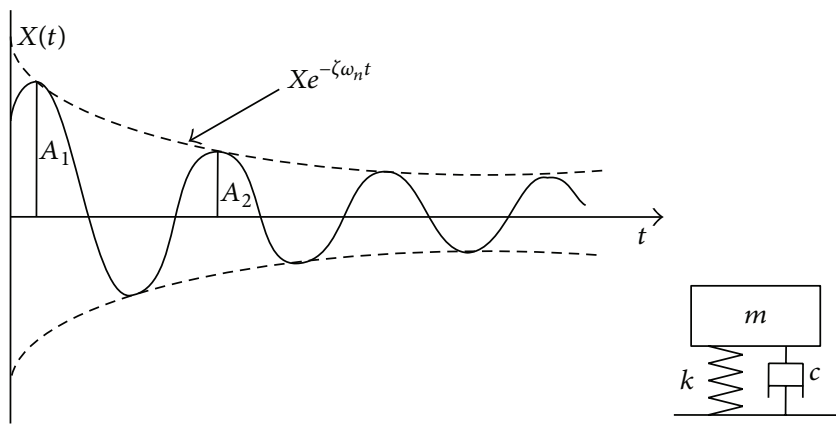

FIGURE 2: The decay curve of a single-degree-of-freedom system after the impact.

peak $\left(A_{1}\right.$ and $A_{2}$ ) corresponds to the decaying sinusoidal function. The logarithmic decrement $\delta$ can be expressed in the following formula:

$$
\delta=\operatorname{In} \frac{A_{1} \sin \left(\omega_{d} t_{1}\right)}{A_{2} \sin \left(\omega_{d} t_{2}\right)} .
$$

The damping coefficient can be found by first calculating the damping ratio $(\zeta)$, which is dependent upon the decrement $(\delta)$ of the system:

$$
\zeta=\frac{1}{\sqrt{1+(2 \pi / \delta)^{2}}} .
$$

The damping coefficient $(c)$ can be found if the mass $(m)$, spring constant $(k)$, and damping ratio $(\zeta)$ are known.

$$
C=2 \zeta \sqrt{m k}
$$

In this study, the stacked corrugated boxes are an MDOF system, the measured decay over a time frame after the impact will neither develop an exact exponential decay function in free vibration as viscous damping illustrated in Figure 2 in a SDOF system nor present a linear decay curve as a damping friction. Therefore, the damping ratio $\zeta$ of the system cannot be directly derived from the formulas (1) to (3) as the ratio of the amplitude of the first-to-second peak may not be the same as the third-to-fourth peak using the that would result in variation in calculation of the damping ratio.

In order to use logarithmic decrement method in an MDOF system, two Butterworth digital filters based on

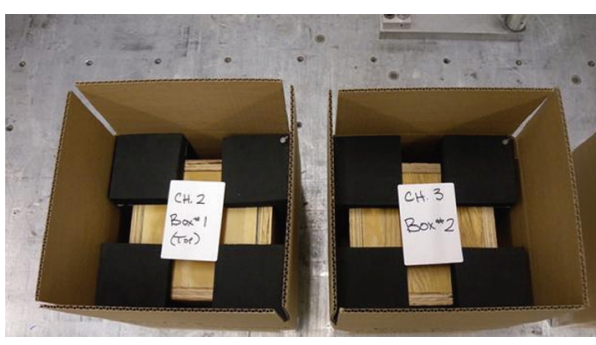

FIGURE 3: Corrugated boxes containing cushion foams and wooden box.

MATLAB were applied to the collected data for separating the viscous damping from the dry friction damping. A sixth-order-low-pass filter was used to eliminate the viscous damping and noise in the system, and a band-pass filter was used to eliminate the dry friction damping and the noise in the system.

2.3. The Curve-Fit Method. Another outcome from the oscilloscope is the PSD spectrum that is calculated based on the collected acceleration. From the PSD spectrum, the natural frequency of each stacked corrugated box $\omega_{n}$ can be identified by the peaks on the PSD spectrum. Using the trend function in the spreadsheet, an approximated trend curve $X e^{-\zeta \omega_{n} t}$ in Figure 2 could be generated easily from the displacement response. With the spreadsheet-generated $X e^{-\zeta \omega_{n} t}$, the damping ratio $\zeta$ can be derived.

2.4. Materials. Corrugated box is made of corrugated board, which is a sandwich structure consisting of two kraft paper faced liners and one medium liner, and the board is regarded as an elastic solid material. The corrugated box selected was a regular slotted container (RSC). RSC code 0201 type A 32 ECT C-flute corrugated paper board was used to construct the corrugated box. The size of the box was $32.48 \mathrm{~cm}(\mathrm{~L}) \times$ $32.48 \mathrm{~cm}(\mathrm{~W}) \times 20.32 \mathrm{~cm}(\mathrm{H})$, and the thickness of the corrugated board was $0.4064 \mathrm{~cm}$ and contained a $6.8 \mathrm{~kg}$ wooden box filled with sand and cushioned in all eight corners with $.085 \mathrm{~kg}$ of the foam. The wooden box and sand are just used as a well-positioned dummy product. The mass of each box, $m$, is the sum of the cardboard box $(0.312 \mathrm{~kg})$, the eight foam corners (.085 kg each), the wooden box $(2.27 \mathrm{~kg})$, and the sand filling the wooden box $(4.54 \mathrm{~kg})$, for a total weight of $7.8 \mathrm{~kg}$ (see Figure 3 ).

The viscous damping of these stacked corrugated boxes mainly comes out of the box structure itself where the air is trapped inside the box and the flute of the corrugated board compresses and decompresses along with the side panel. The dry friction damping occurs in the interfaces between the boxes and the floor. From Figure 1(b), dry friction damping would appear to arise from horizontal motion, and in a single column stacking, horizontal motion is constrained. So, since there is no major shifting, the only dry friction in this study will be the friction between the corner cushion and the inner surface of the corrugated panel that transfers the movements to the flap panel of the corrugated box. 


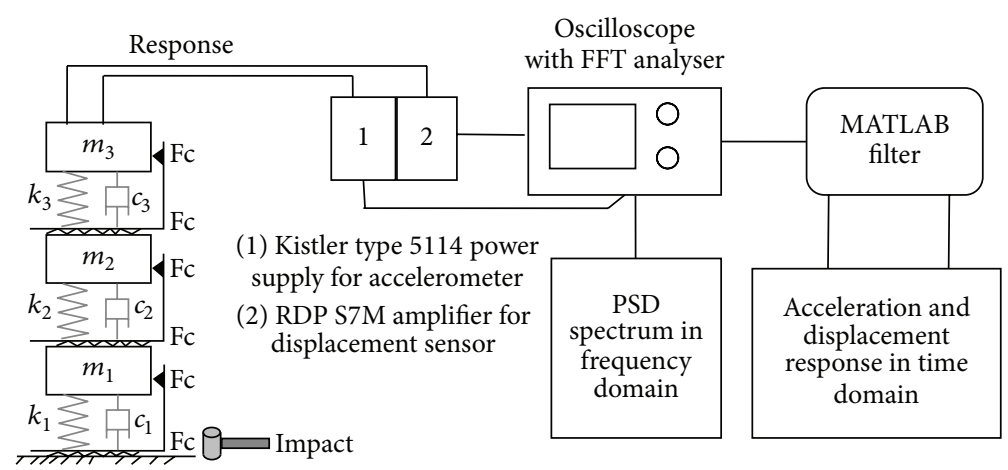

FIgURE 4: Schematic setup of the measurement and calculation of the damping ratios.

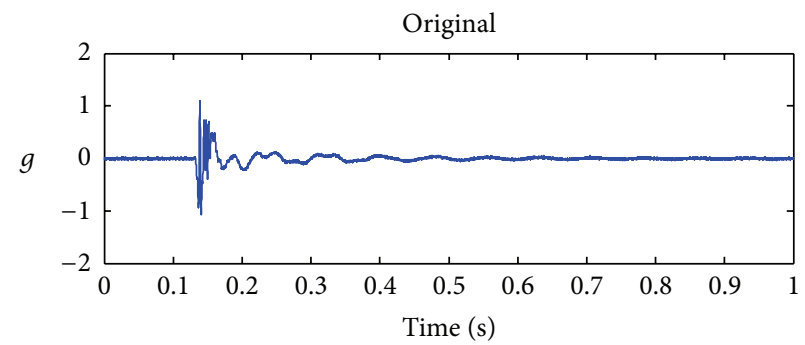

(a)

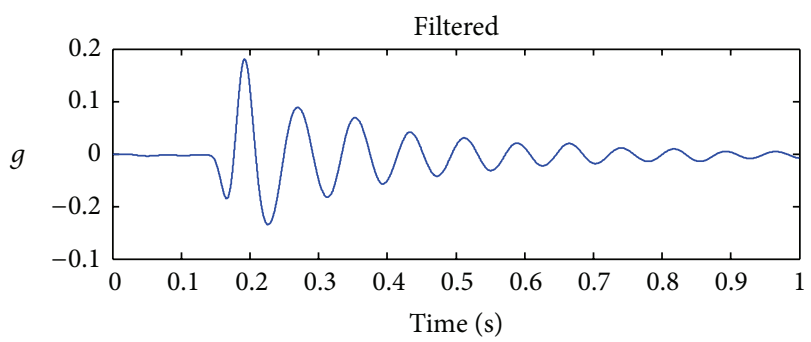

(b)

FIGURE 5: Original acceleration data and with $20 \mathrm{~Hz}$ low-pass filter.

2.5. Measurement. The displacement sensor and accelerometer were initially attached to the top of the corrugated box near the side panel for measurements. The displacement sensor D6 Series LVDT was installed to a clamp holder on a ring stand that is placed on a support structure such as a table, and the pin (sensor) from the LVDT was attached to the top of the box to collect the displacement movement. The pin for the displacement sensor was positioned on the box so that the axis of the displacement sensor would be the same as the hammer strike. The other end of the displacement sensor was connected to an RDP L7M amplifier that connected to the oscilloscope. A Kistler SN 2003532 K-SHEAR8704B50 M1 accelerometer was directly attached to the box using wax for acceleration sampling tests, and the other end was then attached to the oscilloscope. Both displacement and acceleration sensors were placed adjacent to each other, and data were recorded from both sensors simultaneously when the impulse hammer was applied on the bottom of the platform. A Kistler 9722A2000 SN C130699 impulse hammer was used to strike the box. The data was collected on the oscilloscope over a 40millisecond period using the single-sequence function. The PSD spectrum was generated by the oscilloscope itself.

In order to increase the visible amplitude of displacement, the voltage division on the oscilloscope was set to $200 \mathrm{mV}$. Using the single-sequence function on the oscilloscope allowed for both the displacement data and acceleration data to be observed simultaneously after each test was performed. If the acceleration data amplitude was difficult to read, the scaling was increased after the data was collected. Data was assessed quickly to ensure that only a single strike of the hammer was made and that no outside noise had influenced the data. All data gathered on the oscilloscope was saved to a floppy disk and transferred to a spreadsheet and MATLAB for further analysis.

\section{Results and Discussion}

Prior to the collection of the raw data, calibrations were carried out to check the linearity, repeatability, and reciprocity of the system. The linearity check was carried out by exciting the structure with two different input amplitudes to ensure that the shapes of the two response curves are similar, and collected data are independent from excitation amplitudes. A linear structure should yield also an identical response curve, when the testing is repeated in different time intervals, and finally, reciprocity is checked by observing the system response while the impact locations are changed.

The data collected comprises the acceleration, displacement response in time domain, and the FFT calculated PSD spectrum in frequency domain for the 3-layer stacked corrugated boxes.

The original corrected data and filtered data are illustrated in Figures 5-11. Both acceleration and displacement showed clear two decay patterns in time domain. The 1st zone is from the impact to 200 seconds, and the 2 nd zone is from 200 seconds onwards. The 2 nd zone demonstrated a clear dry friction decay pattern where the decay is decayed linear with time, whereas the envelope of the oscillatory motion in the 1st zone decays exponentially with the half-cycle number.

Twenty $\mathrm{Hz}$ was determined as the cutoff for the lowpass filter, as it was the lowest frequency that could be used that did not alter the original shape of the unfiltered data. 


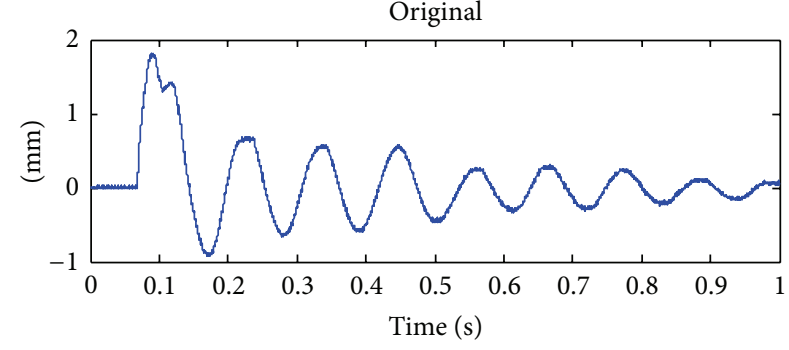

(a)

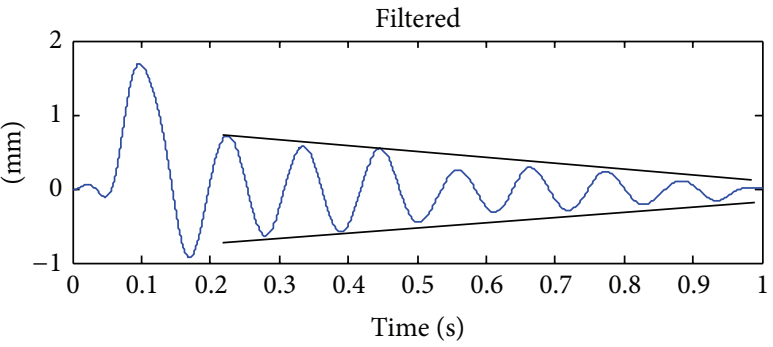

(b)

FIgure 6: Original displacement data and with $20 \mathrm{~Hz}$ filter.

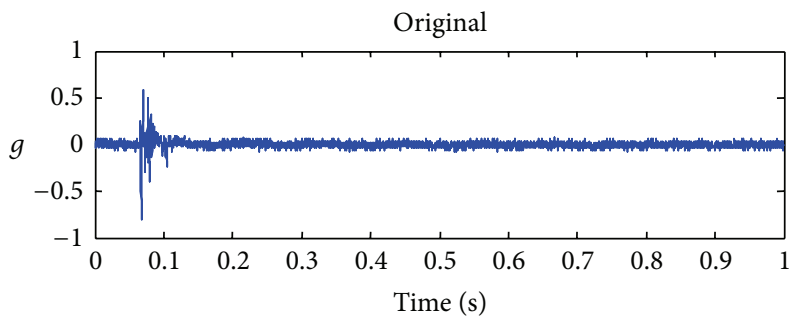

(a)

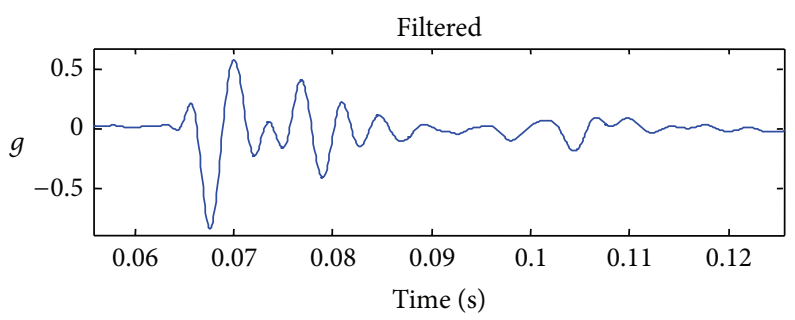

(b)

FIGURE 7: Band-pass filtering of three-box-stacked-corrugated boxes with accelerometer.

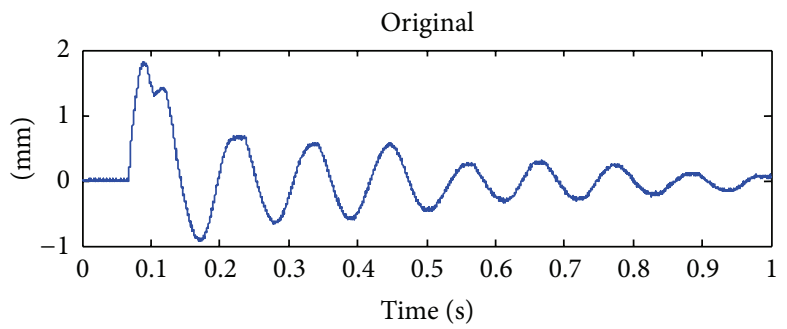

(a)

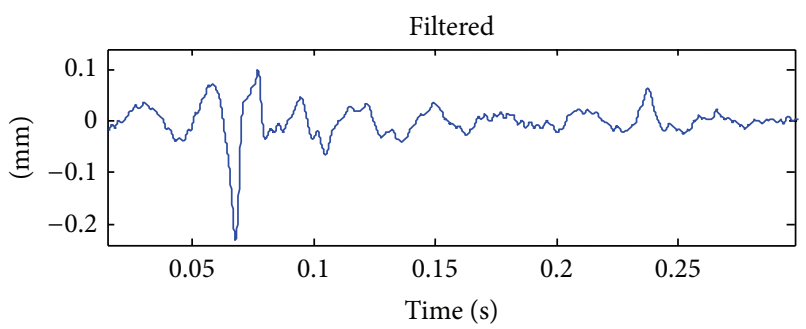

(b)

FIGURE 8: Band-pass filtering of three-box stack with displacement sensor.

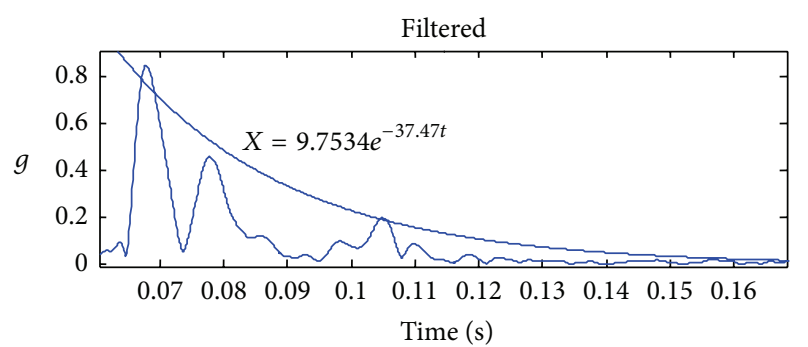

Figure 9: Absolute value of Hilbert transform with decay curve overlaid to show viscous damping decay for accelerometer.

The acceleration data and filtered data for a three-box stack are stated in Figure 11. Straight lines were placed on the graph to show the general linear decrement of the friction damping.

Although viscous and dry friction damping exist across all ranges of frequencies and cannot be practically separated (or attenuated) by means of filters, filters were added to identify the dominated friction type over the responding time when the package system was excited.

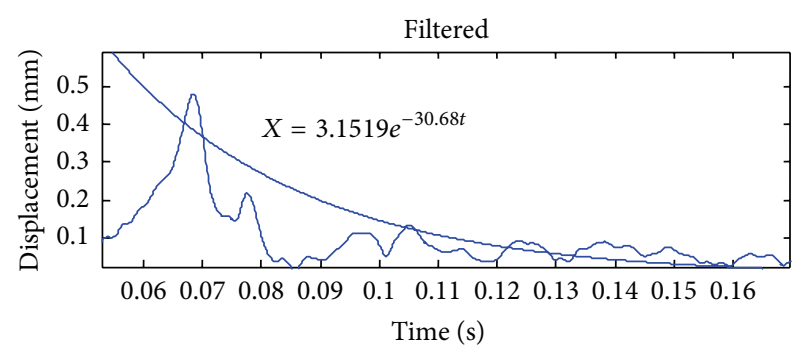

Figure 10: Absolute value of Hilbert transform with decay curve overlaid to show viscous damping for displacement sensor.

Displacement data was filtered with the same $20 \mathrm{~Hz}$ low-pass filter in order to compare the damping ratio derived from the displacement sensor and the accelerometer. The displacement graph and filtered displacement graph are as shown in Figure 6.

The dry friction damping resulting in linear decay is caused by the frictional forces of the box stack. This data should not be used to determine the viscous damping of 
TABLE 1: Damping ratio calculations with low-pass filter.

\begin{tabular}{lcccc}
\hline & $\begin{array}{c}\text { Damping ratio calculations with low-pass filtered data using } \delta=\ln \left(\left(A_{1} \sin \left(\omega_{d} t_{1}\right)\right) /\left(A_{2} \sin \left(\omega_{d} t_{2}\right)\right)\right) \\
\text { Average decrement }\end{array}$ & Minimum decrement & Maximum decrement & Average damping ratio \\
\hline Acceleration data & 0.4644 & 0.0390 & 0.6302 & $\zeta=0.0737$ \\
Displacement data & 0.2147 & 0.2085 & 0.2209 & $\zeta=0.0342$ \\
\hline
\end{tabular}

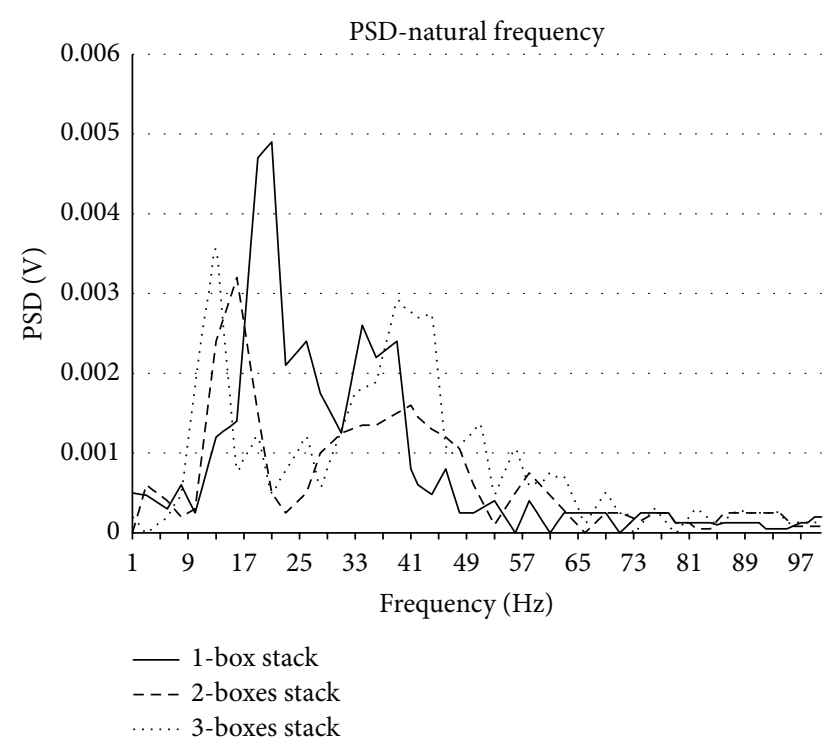

FIGURE 11: Frequency spectrum calculated based on oscilloscope's FFT from one-box stack to three-boxes stack.

the system, and the damping ratio of the system is not determined by the friction in the system.

The band-pass filter was set between $32 \mathrm{~Hz}$ and $450 \mathrm{~Hz}$. Applying the band-pass filter allows the impact signal to catch up when the box is initially struck. The filtered data for acceleration for the three-box test is shown in Figure 7.

The bottom part of Figure 7 is an enlarged filtered view that the signals decay from $7 \mathrm{~ms}$ to $200 \mathrm{~ms}$, representing a close to exponential decay. The behavior of viscous damping of the system is observed. The filtered data for three boxes with a displacement sensor is shown in the figure. Looking at the original data between 0 and 0.1 seconds, there appear to be a few peaks with greater amplitude than the general signals recorded, showing a similar exponential decay curve as the acceleration. Any lower frequency vibrations occurring when data was collected, which were not caused by the impact of the hammer, will not influence the viscous damping curve found after the filter is applied.

As can be seen in both charts of Figure 8-displacement and accelerometer band-pass filtered data-the decay is generally exponential. This result contrasts with the constant decay when a low-pass filter is applied. Also, the curve is entirely contained within the first $200 \mathrm{~ms}$ after the hammer strikes, whereas the low-pass filtered data continues to decay beyond the recording time for the experiment.

3.1. Determine the Damping Ratio Using Logarithmic Decrement Method. Although the logarithmic decrement calculation applies mostly in the scenario of viscous damping, the logarithmic decrement method was used in calculating the damping ratio for both viscous damping and dry friction damping. Only positive decrements were collected after the initial impact had ended. Based on Figures 5 and 6 with the $20 \mathrm{~Hz}$ filter, averaged decrements were calculated from 10 peaks on the decay curve for both acceleration and displacement data. The averages of the decrements, as well as the average damping ratio, are included in Table 1.

Table 2 shows the damping ratio calculation based on Figures 7 and 8 . It is observed that the damping ratio based on displacement is different from the accelerometer. The acceleration of the logarithmic decrement has a very wide deviation. The displacement data demonstrated a relatively consistent value. The damping ratio based on displacement is recommended for a dry friction damping calculation.

When comparing the damping ratio between dry friction and viscous damping, the viscous damping ratio is significantly higher than the dry friction in the multilayerstacked-corrugated box. That ratio is mainly determined by the impacting direction of the hammer and the nature of the system. There is significantly less variation in computed decrements and damping ratios when the friction damping (low frequency data) is filtered out and only the viscous damping curve remains.

3.2. The Curve-Fit Method. The envelope for the band-pass filter was calculated using the absolute value of the Hilbert transform defined in MATLAB. Using the absolute value of the Hilbert transform allows the curve to be more easily realized. The maxima then can be placed in Excel so that a best-fit decay curve can be fit to the original response curve. The chart in Figure 9 shows the curve-fit function based on a three-box test with the accelerometer, and the chart in Figure 10 illustrates the envelope curve fit for the same threebox test using a displacement sensor.

Applying a trend function directly to the response data is a simplified approach. Although the curve fit method appeared to be more adequate than the logarithmic decrement method, the overlaid decay curve in Figure 10 is relatively oversimplified. In the future study, the instantaneous magnitude over the time should be mathematically modelled to extract the damping or friction coefficients based on the damping mechanism. In addition, a high-pass filter is needed to ensure the integrity of the sampled signals for analysis.

The frequency spectrum resulting from applying a Fourier transform to the acceleration data is stated in Figure 11. The first peak frequency seen in the spectra is at $10 \mathrm{~Hz}$. This frequency represents the substructure $c_{1}-k_{1}$ in Figure 4 , and it has a very large magnitude in the graph. The second peak frequency is at $16 \mathrm{~Hz}$. This frequency represents the natural frequency of substructures $c_{2}-k_{2}$ in Figure 4. 
TABLE 2: Damping ratio calculations with band pass filter.

\begin{tabular}{|c|c|c|c|c|}
\hline \multicolumn{5}{|c|}{ Damping ratio calculations with filtered Hilbert transform data $\delta=\ln \left(\left(A_{1} \sin \left(\omega_{d} t_{1}\right)\right) /\left(A_{2} \sin \left(\omega_{d} t_{2}\right)\right)\right)$} \\
\hline & Average decrement & Minimum decrement & Maximum decrement & Average damping ratio \\
\hline Acceleration data & 0.7266 & 0.6158 & 0.8375 & $\zeta=0.1149$ \\
\hline Displacement data & 0.7963 & 0.4801 & 1.1125 & $\zeta=0.1257$ \\
\hline
\end{tabular}

TABLE 3: Damping ratio calculated with the curve-fit method.

\begin{tabular}{lll}
\hline \multicolumn{3}{c}{ Damping ratio calculated with the exponential decay function $X e^{-\zeta \omega_{n} t}$} \\
\hline$X=9.7534 e^{-37.47 t}$ for acceleration & $\omega_{n}=38 \mathrm{~Hz}\left(c_{3}\right.$ and $\left.k_{3}\right)$ & $\zeta=0.157$ \\
$X=9.7534 e^{-37.47 t}$ for acceleration & $\omega_{n}=16 \mathrm{~Hz}\left(c_{2}\right.$ and $\left.k_{2}\right)$ & $\zeta=0.373$ \\
$X=9.7534 e^{-37.47 t}$ for acceleration & $\omega_{n}=10 \mathrm{~Hz}\left(c_{1}\right.$ and $\left.k_{1}\right)$ & $\zeta=0.597$ \\
\hline
\end{tabular}

Comparing the method of logarithmic decrement with the curve-fit method, the logarithmic-decrement-derived damping ratio $(\zeta=0.1257)$ is close to the damping ratio $(\zeta=0.157)$ of the $c_{3}-k_{3}$ structure.

The third peak frequency is between 27 and $38 \mathrm{~Hz}$, representing the $c_{3}-k_{3}$ substructure. Figure 11 shows a summary of FFT analysis for the four measurements that result in a 31-40 Hz natural frequency for a one-box stack and another two peak frequencies, $10 \mathrm{~Hz}$ and $16 \mathrm{~Hz}$, for the two-box stacking structure. The natural frequencies calculated are used previously when calculating the damping ratios using the exponential function derived from the viscous damping decay curve.

Table 3 summarizes the calculated damping ratios based on three different natural frequencies. These damping ratios were determined by placing the natural frequency into the curve-fit formula $X=9.7534 e^{-37.47 t}$ in Figure 9. Given a natural frequency $\omega_{n}$ equal to $38 \mathrm{~Hz}$ for the accelerometer and a damping ratio of 0.157 , a natural frequency of $16 \mathrm{~Hz}$ results in a damping ratio of 0.373 , and finally, a natural frequency of $10 \mathrm{~Hz}$ results in a damping ratio of 0.597.

\section{Conclusion}

The damping properties of stacked corrugated boxes can be determined by using experimental modal analysis (EMA). This simple method opens up a possibility of simulating an MDOF system in the field or warehouse with simple equipment.

In the stacked corrugated box, the band-pass-filtered decay curve demonstrates an exponential curve, representing the viscous damping and contained entirely within the first $200 \mathrm{~ms}$ in the response curve. In contrast, the low-passfiltered data responds at a later time and decays in a linear pattern, representing the dry friction damping from $200 \mathrm{~ms}$ and beyond after the excitation. The damping ratio of the viscous damping is significantly higher than the dry friction in the system due to the nature of the base excitation.

The viscous damping ratios obtained from the curve-fit method and logarithmic method are different. The damping ratios using curve-fit method are higher than the logarithmic method. The difference is possibly due to the inconsistency between the peaks on the decay. Although the logarithmic decrement method is an effective tool to determine the damping ratio of an SDOF system, an MDOF system cannot be simplified with only one damping ratio. Therefore, the curve-fit method is recommended to determine damping ratios for an MDOF system. It is noted that the damping ratio $0.1149-0.1259$ using the logarithmic decrement method is close to the Godshall's [1] recommendation (0.115) for vertical-stacked corrugated boxes.

Theoretically, the damping ratio derived from acceleration and displacement sensors should be identical when the decayed curve is a harmonic movement. This study showed that the damping ratio derived from the accelerometer and displacement sensor is in partial agreement due to the nonlinearity and multidegree of the freedom.

It would be interesting in the future to study dry friction behavior where the roving hammer impact direction is perpendicular to the stacking direction of the corrugated boxes. In this scenario, the dry friction interface between the boxes may play a major role in determining the overall damping properties simulating horizontal impact and vibration of the stacked corrugated boxes.

\section{References}

[1] W. O. Godshall, "Frequency Response, Damping, and Transmissibility Characteristics of Top-Loaded Corrugated Containers," United States Department of Agriculture, Forest Service Research Paper FPL 160, 1971.

[2] W. O. Godshall, "Vibration Transmissibility Characteristics of Corrugated fiberboard," United States Department of Agriculture, Forest Service Research Paper FPL 211, 1973.

[3] T. J. Urbanik, "Transportation vibration effects on unitized corrugated containers," United States Department of Agriculture, Forest Service Research Paper FPL 322, 1978.

[4] Z. Chen, K Saito, and K. Kawaguchi, "Shock response of frictional-viscous damping model for cushioning package," Journal of Applied Packaging Research, vol. 5, no. 4, pp. 197-214, 2011.

[5] J. Wang, Z.-W. Wang, L.-X. Lu, Y. Zhu, and Y.-G. Wang, "Three-dimensional shock spectrum of critical component for nonlinear packaging system," Shock and Vibration, vol. 18, no. 3, pp. 437-445, 2011.

[6] J. Wang, X. Hong, Y. Qian, Z. W. Wang, and L. X. Lu, "Inverse sub-structuring method for multi-coordinate coupled producttransport-system," Packaging Technology and Science, 2013.

[7] K. Peleg, "Frequency response of non-linear single degree-offreedom systems," International Journal of Mechanical Sciences, vol. 21, no. 2, pp. 75-84, 1979. 
[8] C. Ge and D. Goodwin, "Determine natural frequencies of stacked corrugated boxes using a roving hammer," Journal of Applied Packaging Research, vol. 5, no. 3, pp. 145-156, 2011.

[9] K. Peleg, "Impact and vibration testing of shipping containers," Journal of Sound and Vibration, vol. 93, no. 3, pp. 371-388, 1984.

[10] B. J. Schwarz and M. H. Richardson, "Experimental Modal Analysis: CSI Reliability Week," Orlando, Fla, USA, 1999, http:// www.systemplus.co.jp/support/data/techpaper/mescope/tech/ 28.pdf. 


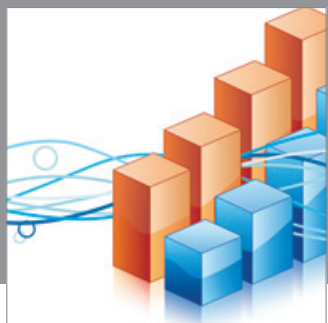

Advances in

Operations Research

mansans

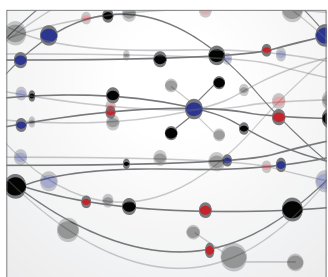

The Scientific World Journal
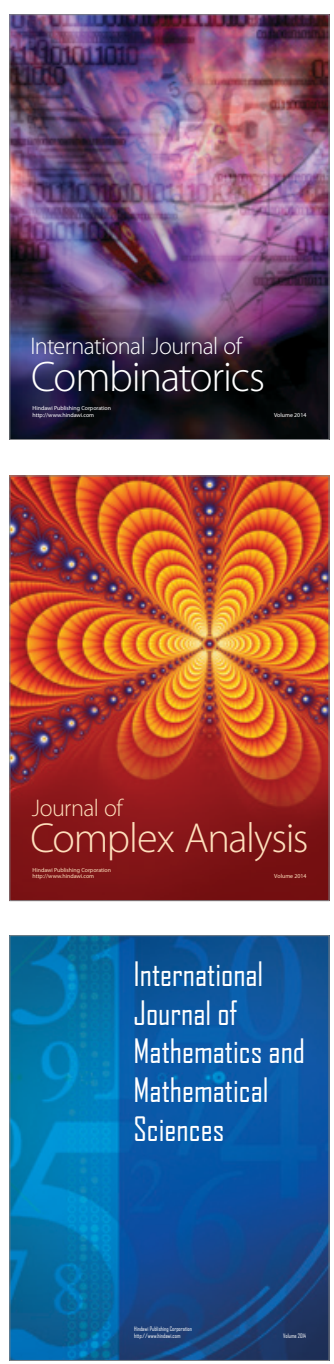
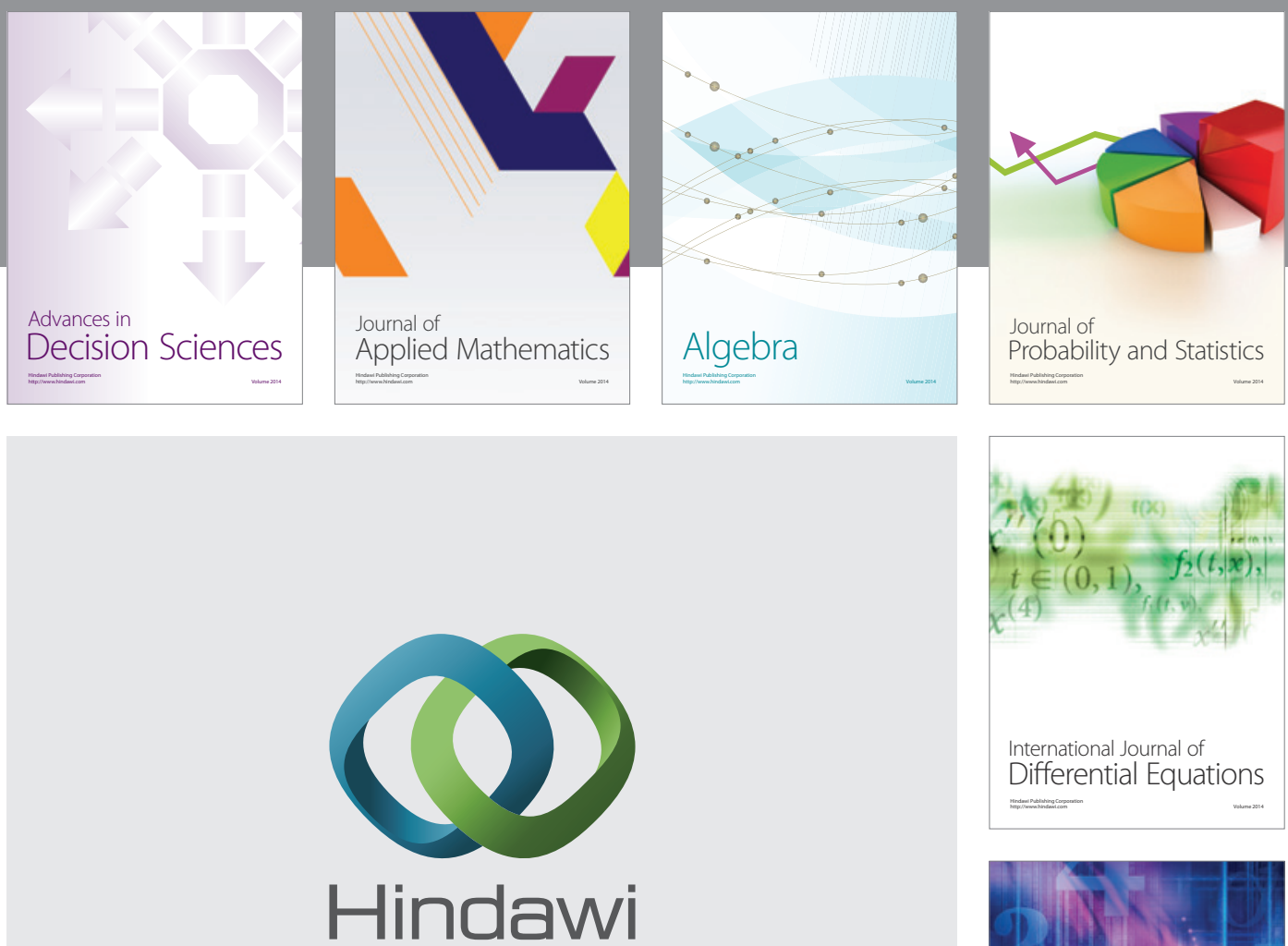

Submit your manuscripts at http://www.hindawi.com
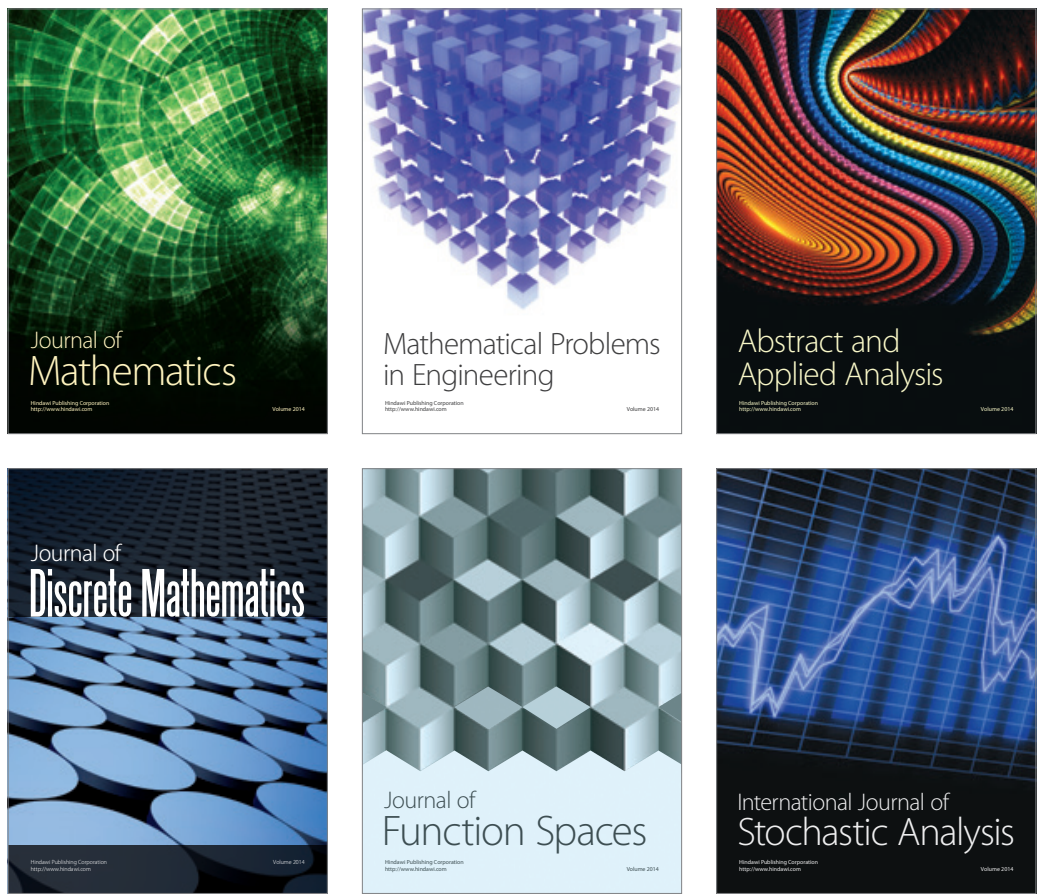

Journal of

Function Spaces

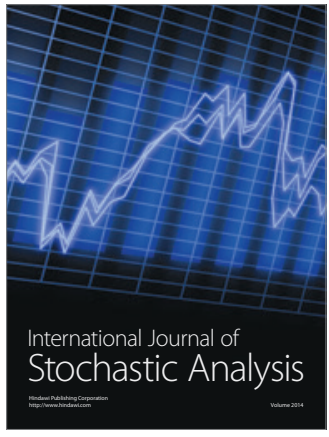

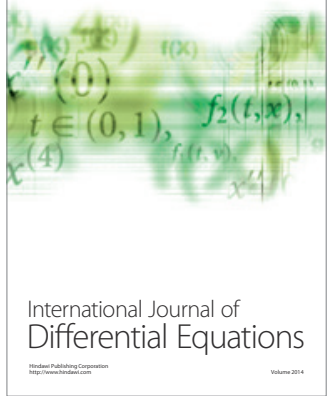
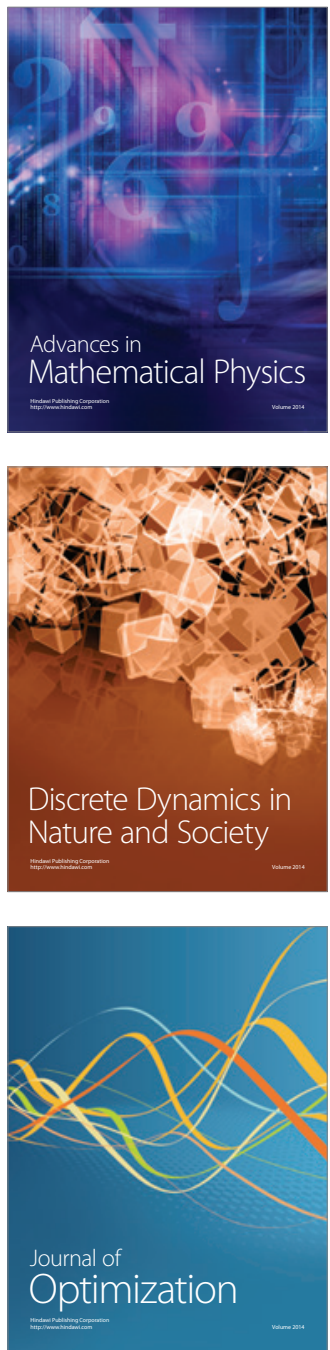